\title{
Revisión del vocabulario español-garífuna sobre salud ${ }^{1}$
}

\section{Marcela Carías ${ }^{2}$}

Universidad Nacional Autónoma de Honduras

\section{RESUMEN}

Estudio de aspectos específicos referidos a procesos de renovación y actualización del garífuna, lengua de la familia arahuaca, hablada en territorios centroamericanos, especialmente de Honduras, Guatemala y Belice. Se centra en el análisis de sus contactos con el español, sobre todo del vocabulario sobre salud.

\section{ABSTRACT}

This study is about specific aspects of the renewal processes in the Garifuna language (belonging to the Arawak family) spoken in Central America, especially in Honduras, Guatemala and Belize. Its contact with Spanish is analyzed particularly regarding vocabulary related to health.

Palabras clave: lenguas indígenas, lenguas amerindias, Centroamérica, garífuna, vocabulario sobre salud.

Keywords: indigenous languages, Amerindian languages, Central America, Garifuna, health-related vocabulary.

${ }^{1}$ Conferencia presentada en el II Simposio Internacional Las lenguas de la Baja Centroamérica y sus vecinos, 2009. Recibido: 10 de febrero de 2009; aceptado: 26 de abril de 2009.

${ }^{2}$ Correo electrónico: marcariashn@yahoo.com 


\section{Presentación}

En este trabajo se presentan algunos avances de un estudio más amplio sobre los procesos de renovación y actualización de la lengua garífuna en cuanto al cambio tecnológico y científico. Voy a centrarme en la consideración de una investigación terminológica realizada para el área de la salud, que condujo a la preparación de vocabulario de próxima publicación. La experiencia de esa investigación dejó un rico material léxico y sobre todo de un número considerable de interrogantes con respecto a los procesos lingüísticos y sociolingüísticos implicados, que requieren de una mayor investigación.

Como asunto de interés está el de explorar con detenimiento las distintas estrategias lingüísticas con que los garínagu responden a los cambios tecnológicos y científicos, y que demuestran tanto el potencial de la lengua como la creatividad lingüística y cultural de sus hablantes. No menos interés despierta considerar la pertinencia del trabajo terminológico, como proyecto de renovación de la lengua, confrontado con la realidad sociolingüística. Por una parte, se exploran las actitudes de los hablantes y de los líderes de las comunidades garínagu al respecto, y por otra, se considerar la utilidad práctica que la producción de vocabularios especializados de este tipo pueda tener en la vida diaria de las personas.

\section{La lengua garífuna}

El garífuna se habla en Honduras, Guatemala y Belice, así como en los Estados Unidos de América. En cuanto al número de hablantes, las cifras varían según las fuentes: estimaciones recientes para Honduras arrojan 98.000 hablantes que habitan en la costa noroeste (atlántica), en los departamentos de Atlántida, Cortés, Colón, Gracias 
a Dios e Islas de la Bahía; desde el Cabo Camarón hasta la frontera con Guatemala ${ }^{3}$.

González ${ }^{4}$ estima que la cantidad de garínagu asentados en los Estados Unidos oscila entre 75.000 y 100.000, aunque no ofrece datos sobre la situación sociolingüística en términos de mantenimiento o reemplazo de la lengua. Su mayor concentración está en Nueva York, seguido por Nueva Orleans, Los Ángeles, Chicago, Miami, y en menor grado, en Washington D.C. y Boston.

\section{Historia}

El garífuna moderno se originó en la isla de Yurúmain (San Vicente, en las Antillas Menores) hace más de seis siglos. Mucho antes de la conquista europea, esta isla estuvo habitada por los igneri, cuya lengua pertenecía al tronco arawak-maipure. Aproximadamente un siglo antes de la llegada de Colón, contingentes Caribe (hablantes de kalínagu) provenientes del Orinoco migraron hacia las islas, conquistaron a los igneri-arawak, mataron a los hombres y tomaron a las mujeres, quienes transmitían su propia lengua a los hijos. En estos sucesos se basa la explicación de la «asombrosa» característica del habla de esta isla: hombres y mujeres hablan lenguas diferentes (documentada en testimonios del siglo XVII, en especial Breton, Rochefort y Labat). En cualquier caso, tras una etapa de bilingüismo arawak-caribe, los hijos de estas uniones fueron adoptando el arawak como lengua materna.

En el siglo XVII, ingleses, franceses y holandeses se asentaron en algunas islas de las Antillas Menores, expulsando y aniquilando la población aborigen. A fines de ese siglo sólo quedaban residuos de la población caribe-arawak en la isla Dominica y en la de San Vicente (Yurúmain). En 1675, un barco con esclavos africanos naufragó cerca

\footnotetext{
${ }^{3}$ Basado en datos de M. Paul Lewis (Ed.), Ethnologue: Languages of the World, 16a. ed. (Dallas, Texas: SIL International, 2009). Versión en línea: <http://www.ethnologue.com/>.

${ }^{4}$ Nancie L. González, Peregrinos del Caribe. Etnogénesis y etnohistoria de los garífunas (Tegucigalpa: Guaymuras, 2006).
} 
de esta isla, lo que dio lugar a muchas uniones entre africanos y «island caribs», como eran conocidos entonces. De estas uniones surgieron los negros caribe (o «black caribs»), quienes aliados con los franceses se opusieron a los asentamientos ingleses. No obstante, en 1797, los franceses los expulsaron hacia la isla de Roatán frente a la costa norte hondureña; ahí arribaron 2.026 garínagu el 12 de abril de ese año.

La lengua garífuna ha sido considerada un creole por muchos estudiosos, si bien no hay unanimidad al respecto; en tal sentido se suelen aportar las siguientes estadísticas con respecto a su «composición léxica»: 45\% arawak, 25\% kalina (caribe), 15\% francés, $10 \%$ inglés y $5 \%$ español. Además, la gramática y el léxico garínagu son principalmente de origen arawak-maipure (el garífuna es una lengua aglutinante que presenta diferentes secuencias de constituyentes en los niveles morfológico y sintáctico).

Según Anthony Grant ${ }^{5}$, el garífuna moderno es una lengua en la que «la mayor parte del léxico y morfosintaxis son de $\mathrm{A}$, mucho léxico básico y alguno menos básico pertenece a $\mathrm{B}$, y además presenta estructuras sintácticas adicionales y bastante léxico cultural proveniente de $\mathrm{C} \gg$. Explica que $\mathrm{A}$ es una lengua arawak relacionada pero no idéntica con el arawak propio y el taíno, y cuya versión del siglo XVII era el «Island Carib» de Dominica; B es kalina, y C es el francés creole de Dominica. Agrega que el garífuna también cuenta con importante material léxico proveniente del inglés creole y del español.

Por otra parte, no se ha explorado suficientemente la influencia que lenguas africanas, en especial el bantú, puedan haber tenido en el desarrollo del garífuna; así como el asunto de la influencia que haya podido recibir de las lenguas creole del Caribe (especialmente en cuanto a las variantes léxicas del francés utilizadas en las Antillas menores y Haití, y el léxico de creole inglés).

5 Anthony P. Grant, «Contact-Induced Change and the Openness of 'Closed' Morphological Systems: Some Cases from Native America», Journal of language contact - THEMA II (2008), <www. jlc-journal.org>. 
LETRAS 45 (2009) Carías / Revisión del vocabulario español-garífuna

\section{Política lingüística}

En 1997 se adoptó la «Declaratoria de política lingüística para la nación garífuna», a instancias del National Garifuna Council (NGC) de Belice. Este extenso documento abarca todos los aspectos de la planificación lingüística; citaré aquí algunos párrafos:

Nosotros, el pueblo garífuna, aunque vivimos dentro de las fronteras de distintos países (incluyendo, pero no sólo, en Belice, Guatemala, Honduras, Nicaragua, St. Vincent, y los Estados Unidos), representamos una comunidad étnica única y unida, conocida como Nación Garífuna. Nuestra lengua es la garífuna. Esta lengua tiene un diccionario, una ortografía, y un corpus de bibliografía sobre su gramática.

Más adelante, en el apartado correspondiente a «Política de mantenimiento lingüístico», bajo el subtítulo de «Ampliación del léxico», se lee lo siguiente:

El garífuna es una lengua moderna, capaz de expresar la gama completa de intenciones comunicativas de los seres humanos. Nuestra lengua es también capaz de la ampliación léxica hacia campos semánticos especializados de la técnica y la ciencia contemporáneas, incluyendo las matemáticas, las tecnologías de la información, la economía, la política, etc. Por lo anterior, decretamos la conformación de una comisión especial garífuna, con carácter internacional, para que desarrolle y determine nuevo vocabulario apropiado para que los garínagu puedan hablar sobre cualquier materia sin tener que recurrir al uso de lexemas foráneos. Este comité representará a los garínagu de todas las regiones geográficas, y sostendrá 
reuniones regulares en el marco de un proceso de desarrollo de nuevo vocabulario, cuando éste sea necesario ${ }^{6}$.

Otro hecho notable sobre la planificación del status de la lengua garífuna, es su proclamación como «Obra maestra del patrimonio oral e intangible de la humanidad», por parte de UNESCO en mayo de 2001.

Así pues, los garínagu hacen esfuerzos desde los sectores oficiales, privados, no gubernamentales y políticos, para preservar su patrimonio lingüístico y evitar su reemplazo. En los Estados Unidos, tales campañas son importantes y cuentan con apoyo de fundaciones y organizaciones con sede en ese país. En Honduras, por su parte, existe un programa estatal de educación bilingüe e intercultural (PRONEEAAH) desde hace más de tres lustros, pero no ha logrado satisfacer las demandas de las distintas comunidades lingüísticas del país, debido en gran medida a su modelo vertical y a hallarse sujeto a los vaivenes políticos de los gobiernos de turno.

La situación sociolingüística de los garínagu en Honduras no ha sido estudiada a profundidad; algunos datos generales preliminares basados en visitas a la región, inducen a pensar que en muchas comunidades garínagu aledañas a las ciudades de La Ceiba y Tela, el proceso de reemplazo por el español está muy avanzado; mientras en las comunidades del departamento de Colón se evidencia mayor tendencia al mantenimiento.

\section{Proyecto: Vocabularios multilingües en lenguas amerindias}

El proyecto Vocabularios multilingües en lenguas amerindias, auspiciado por la Unión Latina mediante su Dirección de Terminología e Industrias de la Lengua, se ha venido desarrollando

${ }^{6}$ Geneva Langworthy trata detalladamente la política lingüística garífuna en G. Langworthy, «Language Planning in a Trans-National Speech Community» en Indigenous Languages Across the Community, Barbara Burnaby y Jon Reyhner (Eds.) (Flagstaff: Universidad de Northern Arizona, 2002). 
hace unos años en América Latina y se ha publicado ya un Léxico multilingüe (español - quechua - aymara - guaraní - portugués) en 2005, bajo la dirección de Daniel Prado-, que comprende los ámbitos de agroalimentación, biodiversidad y sanidad. En 2008 la Unión Latina impulsó un proyecto similar en Centroamérica, con lenguas amerindias habladas en la región (una por país), sobre el área de la salud. Fui asignada para coordinar el equipo que trabajaría con la lengua garífuna en Honduras, en el que también participaron Teresa Leyva y Carolina David. El objetivo del proyecto fue «constituir un banco de datos terminológicos en diversas lenguas amerindias con el propósito de responder a las necesidades de las comunidades lingüísticas correspondientes». Entre sus características generales se puede citar que la lengua de partida fue el español, los términos por traducir llegaron a 407, y su difusión una vez concluido se haría por Internet, disco compacto e impresión en papel.

Los fundamentos y justificación de este proyecto se enmarcan en dos presupuestos básicos de la terminología: a. «para la traducción, la interpretación y la redacción técnica, la terminología es un conjunto de unidades de comunicación, útiles y prácticas, cuya evaluación se mide en función de criterios de equivalencia, adecuación, precisión y economía; y b. para la planificación lingüística, la terminología es un ámbito del lenguaje donde hay que intervenir para reafirmar la existencia, la utilidad y la pervivencia de una lengua como medio de expresión» ${ }^{7}$.

La metodología fue de corte onomasiológico, con la que se parte del concepto hasta llegar a las designaciones, las realizaciones lingüísticas en una o más lenguas. $\mathrm{El}$ instrumento de trabajo proporcionado, fue un sistema informático de registro de la base de datos, con 407 entradas y sus respectivas acepciones (mayormente tomadas del DRAE). La recolección de datos se realizó en febrero y

${ }^{7}$ M. T. Cabré, La terminología: presentación y comunicación (Barcelona: IULA, UPF, 1999) 69-173. 
marzo de 2008, en Trujillo (Cristales, Santa Fé, Limón, Guadalupe) y en Tegucigalpa.

Este proyecto terminológico contribuye a elevar el status de las lenguas indígenas, a la vez que ofrece herramientas para las cada vez mayores necesidades de la comunicación especializada y la ampliación léxica ya mencionada en la Declaratoria de política lingüística de los garínagu. No obstante, la investigación in situ ha demostrado que un trabajo de esta naturaleza (en términos de sus objetivos) puede verse obstaculizado por factores tales como: a. la situación sociolingüística particular, que en el caso de los garínagu es la de hablantes bilingües en un contexto diglósico, con lo que es usual la alternancia de códigos para situaciones o tópicos específicos; b. las normas pragmáticas características de la lengua (garífuna en este caso), que se manifiestan en estrategias comunicativas concretas y en fenómenos como el tabú lingüístico, entre otros; y c. la presunción de que los «conceptos», en este caso explícitos en el DRAE, puedan revestir cierto carácter de universalidad, sin tener en cuenta los particulares modelos de construcción cognitiva inherentes a cada lengua.

\section{Fenómenos de transferencia léxica observados en el vocabulario español-garífuna, salud (EGS)}

En esta sección se examinarán algunos casos de transferencia léxica del vocabulario EGS. Se ha preferido el término transferencia $^{8}$ a los de interferencia, préstamo y calco, porque refleja mejor el dinamismo inherente a los procesos en situaciones diglósicas o de contacto de lenguas. Es preciso notar que la lengua garífuna presenta una larga historia de transferencia léxica, especialmente de unidades provenientes del kalina, del francés, del inglés y del español,

${ }^{8}$ Introducido por M. Clyne y desarrollado posteriormente por C. Silva-Corvalán, «Transferencia: la influencia que una lengua ejerce sobre otra y, concretamente, el uso en una lengua $\mathrm{B}$ de un rasgo característico de la lengua A». Moreno Fernández (1998). 
totalmente consolidadas en su acervo patrimonial. En la actualidad, las comunidades de habla garínagu mantienen en contacto con el español en Honduras, con el inglés kriol en Belice y con el inglés en los Estados Unidos de América, de tal manera que cada una de estas situaciones sociolingüísticas generará fenómenos particulares y diferenciados.

Para este trabajo, se considera la variedad hondureña del garífuna, pues tanto las fuentes secundarias como primarias utilizadas corresponden a la misma. Al respecto, la dialectología garífuna en Honduras no ha sido suficientemente desarrollada, aunque de manera general se identifican dos variantes dialectales importantes: el garífuna oriental y el garífuna occidental. El oriental se ha ubicado en las comunidades garínagu del departamento de Colón en Honduras y en las de Nicaragua, y como sus características salientes se cita la elisión de /r/ intervocálica, de /-n/ final de palabra, así como la tendencia a utilizar las formas acortadas de las palabras. El dialecto occidental se ubica en los departamentos hondureños de Atlántida, Cortés e Islas de la Bahía; aparte de los contrastes correspondientes a los rasgos mencionados para la variante oriental, no he encontrado una caracterización más detallada del mismo. A esto habría que sumar las variantes de Guatemala y Belize, así como la de los Estados Unidos.

Con relación a las fuentes utilizadas para este trabajo, la elaboración del vocabulario EGS se basó casi en un $50 \%$ en Hererun Wagüchagu (2006, diccionario trilingüe: garífuna, español, inglés). Esta publicación apareció en La Ceiba, límite entre las dos zonas dialectales descritas, con un afán estandarizante, y aunque no hay mención específica sobre la metodología utilizada, está en gran medida basada en diccionarios o vocabularios anteriores (los de Cayetano y Suazo, entre otros). Las principales colaboradoras, o fuentes primarias, para este vocabulario, son originarias y viven en Cristales y Guadalupe, comunidades aledañas a la ciudad de Trujillo (dialecto oriental). Seguiremos para el análisis de los casos propuestos 
a Carmen Silva-Corvalán en su tipología de este fenómeno lingüístico 9 .

A. Primer caso de análisis: Términos muy productivos en garífuna, que frecuentemente aparecen «colocados» en expresiones lexicalizadas, que presentan gran flexibilidad al combinarse con otras unidades y así permiten la creación de nueva terminología. En este primer caso se puede hablar de calco semántico, tanto en el sentido de creación como en el de extensión, puesto que se produce nuevas translaciones a la vez que se amplía el significado de unidades léxicas ya existentes en la lengua, igual que se extiende su campo referencial. Ejemplos:

\section{1. sandi}

\begin{tabular}{|l|l|l|l|}
\hline Español & Garífuna & $\begin{array}{l}\text { En Hererun } \\
\text { Wagüchagu }\end{array}$ & Fuente primaria (oral) \\
\hline Enfermedad & $\begin{array}{l}\text { sandi } \\
\text { (fr. santé) }\end{array}$ & & \\
\hline
\end{tabular}

${ }^{9}$ Transferencia directa: a. Sustitución de una forma de la lengua B (en nuestro caso el garífuna) por una forma de la lengua A (español); b. la incorporación de una forma de $\mathrm{A}$, inexistente en $\mathrm{B}$, sobre todo para designar un objeto o realidad nuevos; y c. la incorporación del significado de una forma de la lengua $\mathrm{A}$ al de una forma existente en la lengua $\mathrm{B}$. Con respecto al último tipo, conocido también como calco semántico, Einar Haugen propone una división en creaciones y extensiones; las primeras serían las translaciones nuevas en la lengua $\mathrm{B}$, mientras las segundas operan a través de la ampliación del significado de una unidad léxica ya existente en la lengua B. Transferencia indirecta: Aumento de la frecuencia de una forma en $\mathrm{B}$, por corresponderse con una categórica o mayoritaria en la lengua A.

Por otra parte, Shana Poplack distingue entre préstamos consolidados, cuando su frecuencia de ocurrencia es alta y son generalmente aceptados; o en transición, cuando están en proceso de difusión. Según Moreno Fernández, la integración de la transferencia léxica en la lengua puede darse: a. manteniendo la fonética y morfología de la lengua influyente; $b$. adaptando la fonética a la receptora; o c. adaptando la morfología y la fonética a la lengua receptora. 


\begin{tabular}{|l|l|l|l|}
\hline Conjuntivitis & & lisandi agu & \\
\hline $\begin{array}{l}\text { Hongo del } \\
\text { maíz }\end{array}$ & lisandi awasi & \\
\hline Adenopatía & & $\begin{array}{l}\text { lisandi íhemeni } \\
\text { (íhemeni: ganglio linfático) }\end{array}$ \\
\hline Cirrosis & & $\begin{array}{l}\text { lisandi ubanaü } \\
\text { (ubanaü: hígado) }\end{array}$ \\
\hline Dermatosis & & $\begin{array}{l}\text { lisandi uroü } \\
\text { (uroü: piel) }\end{array}$ \\
\hline Epidemia & & $\begin{array}{l}\text { burugua sandi } \\
\text { (burugua: } \text { extender, diseminar) }\end{array}$ \\
\hline VIH-SIDA & & $\begin{array}{l}\text { luriba sandi } \\
\text { (luriba: el mal) }\end{array}$ \\
\hline
\end{tabular}

\section{2. árani}

\begin{tabular}{|l|l|l|l|}
\hline Español & Garífuna & $\begin{array}{l}\text { En Hererun } \\
\text { Wagüchagu }\end{array}$ & Fuente primaria (oral) \\
\hline $\begin{array}{l}\text { Medicina, } \\
\text { remedio }\end{array}$ & árani & & lárani infeksion \\
\hline Antibiótico & & & $\begin{array}{l}\text { lárani líñünün anigi } \\
\text { (línüñün anigi: asma) }\end{array}$ \\
\hline Antiasmático & & $\begin{array}{l}\text { lárani gáriti } \\
\text { (gáriti: dolor) }\end{array}$ \\
\hline Analgésico & & $\begin{array}{l}\text { lárani idiheri } \\
\text { (idiheri: } \text { nerviosismo, ansiedad) }\end{array}$ \\
\hline Calmante & & & \multicolumn{2}{|l}{} \\
\hline
\end{tabular}

Así mismo, se puede mencionar dentro de este primer caso, 'ilu' (hinchazón, hidropesía); ejemplos (fuente oral): lilu agülerü (faringitis), lilu urageirugu (gastritis) y liludü ílagülei (flebitis).

B. Segundo caso de análisis: Calcos semánticos en el sentido de nuevas translaciones a partir de los recursos existentes en la propia lengua; ejemplos: 
1. cráneo:

2. cuero cabelludo:

3. cefalea:

4. artritis:

5. hipertensión: abu ichügü (oral)

uraü ichügü (oral)

ligari ichügü (oral)

ligari abu (oral)

liñun hitaü $(\mathrm{HW})$

El término 'ichügü' (cabeza), al igual que en muchas lenguas, en garífuna presenta un potencial metafórico importante, tal como se evidencia en: 'lichügü uri' (pezón), 'lichügüdina' (capital del país), 'lichügü egi' (cabeza de rallador de yuca), 'lichügü yawe' (llave de ducha), lichú aau (sabiduría), entre otros (todos estos ejemplos tomados de $\mathrm{HW})^{10}$. Los ejemplos (3) y (4) se forman a partir de 'gáriti' (dolor, dolencia), frecuente en el ámbito de la salud, aunque los demás casos encontrados no muestran lexicalización y no fueron propuestos como nuevos términos por las fuentes orales: 'ligari ari' (dolor de muelas), 'ligari arigeirugu' (de oídos), 'ligari urageirugu' (de estómago), etc. El ejemplo (5), incluido en el diccionario HW y por lo tanto ya consolidado, se formó a partir de 'íñuni' (altura) y 'hitaü' (sangre) ${ }^{11}$.

C. Tercer caso de análisis: Calcos semánticos manifiestos en la forma de soluciones perifrásticas, con mayor o menor grado de consolidación en el sistema; de las mencionadas en estos ejemplos, solamente la primera aparece en el diccionario HW.

${ }^{10}$ A. Constenla Umaña (en «Construcción posesiva y economía morfoléxica en las lenguas del área colombiano-centroamericana», Lingüística Chibcha XI (1992): 101-114) argumenta que en casos como este no se puede hablar de expresiones metafóricas y aduce que «el carácter metafórico se debe sin duda a la consideración de que una de las traducciones a la lengua del investigador es la básica». Este autor concluye que «una solución más razonable es pensar que el significado de elementos como /tai:ki/ o /tuki:/ sea el común a todas sus apariciones y no el de un entorno en particular» (pp. 110-111).

11 Otro ejemplo de calco en el sentido de nueva traslación, que aparece en el diccionario HW, es 'liñun-agei lúrudu' para Corte Suprema de Justicia (altodepósito-de leyes; 'lúrudu' del fr. loi du roi). 
Ejemplos:

\begin{tabular}{|l|l|l|l|}
\hline Español & Garífuna & Traducción literal & Fuente \\
\hline Cesárea & agüriahani lau biti & parto - con la - herida & HW \\
\hline Clínica & lubiñe surusia & en la casa de - doctor & Oral \\
\hline Cálculo & $\begin{array}{l}\text { dübü lidan lubana / } \\
\text { kidüni }\end{array}$ & $\begin{array}{l}\text { piedra }- \text { en la/el }- \\
\text { vesícula / riñón }\end{array}$ & Oral \\
\hline Colesterol & agülei lida hitaü & grasa - en la - sangre & Oral \\
\hline Embrión & $\begin{array}{l}\text { irahü turagairügüli } \\
\text { lüguchü* }\end{array}$ & $\begin{array}{l}\text { hijo(a) - en el interior } \\
\text { de ella }- \text { la madre }\end{array}$ & Oral \\
\hline Hematoma & $\begin{array}{l}\text { moróu lida(n) } \\
\text { turaü** }\end{array}$ & morado - en su - piel & Oral \\
\hline Hidrocefalia & duna lida(n) ichügü & agua - en el - cerebro & Oral \\
\hline
\end{tabular}

* Se registra también: 'daga' (feto, HW).

** Se registra también 'makü' (morete, HW).

D. Cuarto caso de análisis: Transferencias directas, para designar un objeto o realidad nuevos. Para ilustrar los tipos de integración de las transferencias que se dan en la lengua garífuna, se ha incluido ejemplos tanto de préstamos históricos plenamente consolidados en el sistema, como de aquellos que están en transición; con ello se puede apreciar que las transferencias integradas históricamente a la lengua garífuna, presentan una marcada adaptación morfológica y fonética; mientras que las más recientes se adoptan como «préstamos puros» (en términos de E. Haugen), conservando la forma y significado de la lengua original.

Para este último caso conviene una aclaración en cuanto a la perspectiva del estudio de situaciones comunicativas en comunidades diglósicas, especialmente en lo que se refiere al fenómeno conocido como cambio de código ${ }^{12}$ (code-switching). La información de campo recabada y las observaciones realizadas durante el desarrollo de este

${ }^{12}$ Cambio de código o alternancia de código, es una estrategia comunicativa de los hablantes bilingües, que consiste en el uso alterno de dos lenguas en la misma frase o enunciado. 
proyecto, permiten concluir lo siguiente:

a. Los garínagu utilizan el español cuando acuden a los centros de salud, clínicas, hospitales, etc.

b. Cuando hablan en garífuna sobre salud, y requieren utilizar términos o expresiones especializados referidos particularmente a nombres de enfermedades o tecnología médica novedosa, eligen cambiar de código hacia el español, atendiendo a la morfosintaxis y fonética de esta lengua, para después regresar al garífuna nuevamente. Se trata de hablantes bilingües con una buena competencia comunicativa en ambas lenguas, así como con un reconocimiento de la diferenciación de funciones típica de situaciones diglósicas.

c. La lengua garífuna posee una gran cantidad de recursos para hablar sobre la salud y la enfermedad, como queda evidenciado en este trabajo (únicamente un 13\% del total de las 407 entradas fueron aportadas como «neologismos» por las colaboradoras del proyecto). Valga comentar que ellas, al ser consultadas sobre los equivalentes en garífuna de términos tales como 'dispepsia' o 'escarlatina', me preguntaron, en primer lugar, si yo suelo utilizar esas palabras o si conozco su significado exacto en español; y en segundo lugar, dijeron que no merecían el esfuerzo de buscar soluciones perifrásticas para adoptarlas en su lengua, dado que si las necesitan usar, recurrirían a la forma española.

d. Jan-Peter Blom y John Gumperz, en su estudio sobre el cambio de código en Noruega ${ }^{13}$, distinguen dos tipos: situacional y metafórico; el primero relacionado estrechamente con la situación comunicativa y sus normas y condicionantes sociales, y el segundo con tipos particulares de temas más que con un cambio en la situación social. Este cambio de código metafórico se observa en los hablantes garínagu en contextos cuyo tópico es la enfermedad, y en este trabajo se manifiesta en la existencia de parejas de términos (sinónimos) en las que uno de ellos está marcado semánticamente como perteneciente

${ }^{13}$ J. P. Blom y J. Gumperz. «Social Meaning in Linguistic Structure: Code-

Switching in Norway», The Bilingualism Reader (Londres: Routledge, 2000). 
al imaginario garífuna, mientras el otro es relativamente más neutro. En los ejemplos que siguen, se aprecia que el término neutro suele ser una transferencia, si bien en muchos casos plenamente consolidada. Ello podría asociarse con la construcción y reproducción de los imaginarios colectivos de la enfermedad, en este caso distintos pero encontrados en el contacto lingüístico cotidiano.

\section{Transferencias consolidadas (HW)}

Del español:

abakunara

abufida

balúsamü

güripe

konhuntiwitisi

meskinu

operararü

patu

sesu

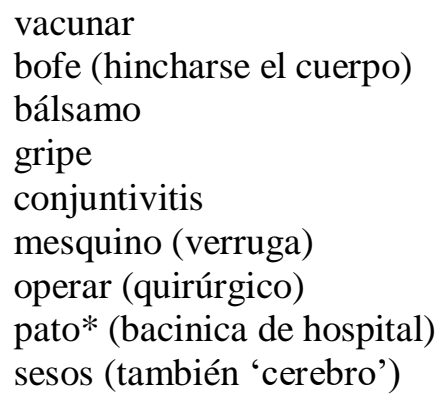

Del francés:

abuleiseira

adureida

blesser (golpearse, lastimarse)

diwéin étroit (adelgazar)

fuñei

úmari

lifidali

du vin (vino)

poignet (puño, muñeca)

mari (marido)

l'hôpital (hospital)

chirurgien (médico)

Del inglés:

wounu

háspitali

wound (herida)

hospital

míligi

péisini

pili

milk (leche)

poison (veneno)

pill (pastilla)

yómani

young man (hombre joven) 
saisi size (tamaño, talla)

kuantatí quantity (cantidad)

ifingaü finger (dedo)

\section{Aportaciones de las colaboradoras (préstamos «puros»o íntegros, del español)}

apendicitis, arterioesclerosis, aspirina, atrofia, bronquitis, cistitis, colirio, deshidratación, difteria, dispepsia, embolia, encefalitis, enfisema, flema, gárgara, gastroenteritis, gota, hernia, homeopatía, hormona; y con adaptaciones fonéticas: fluhu, tetanu, forse, gangliü

\section{Sinónimos}

\begin{tabular}{|c|c|c|}
\hline Español & Garífuna & Garífuna \\
\hline & & estoubugo (esp. estado) \\
\hline engripado & yadünuti(tu) & kustipóu (esp. constipado) \\
\hline tartamudez & dagádagaü & lougua tariñagun \\
\hline hospital & luban sandi & háspitali (ingl.) \\
\hline & & lifidali (fr. l'hôpital) \\
\hline cáncer & bésuru* & kánseru \\
\hline desmayo & óunwehani $* *$ & falalaü \\
\hline cerebro & ichügü & sesu \\
\hline conjuntivitis & figigu & konhuntiwitisi \\
\hline riñón & íhuaraü & kidüni (ingl. kidney) \\
\hline enano & fedé & hinanu \\
\hline calambre & ederehani & karanpu (ingl. cramp) \\
\hline & igañi & hénio \\
\hline herida & biti & wounu (ingl.) \\
\hline VIH-SIDA & luriba sandi & gádürü-leta \\
\hline
\end{tabular}

* 'bésuru' (cáncer) aparece registrado en el diccionario garífuna publicado en la página de Internet del Garifuna Institute, con base en Los Angeles, California. En HW no aparece este término en particular, pero sí 'bésun': herida que carcome hasta hacerse grande.

** 'óunwehani' aparece en la entrada correspondiente al español 'desmayo'; sin embargo, se hace la siguiente acotación: «pérdida de la conciencia durante la 
LETRAS 45 (2009) Carías / Revisión del vocabulario español-garífuna

ceremonia dügü».

Para finalizar con este análisis, pasemos a comentar el último ejemplo citado en la lista precedente:

VIH-SIDA: No aparece en HW

1. luriba(n) sandi (aportado por Carolina David)

luriban: maldad, lo malo, lo prohibido (HW).

sandi: enfermedad

Se asocia con lo malévolo y con lo impuro.

\section{2. gádürü-leta}

(cuatro-letras)

Este término aparece registrado en dos fuentes escritas: el citado diccionario del sitio de Internet del Garifuna Institute; y en la Ley de igualdad de oportunidades para la mujer, traducida al garífuna y publicada por el Instituto Nacional de la Mujer, Tegucigalpa, 2004. Valga aclarar que ninguna de estas fuentes menciona 'luriba sandi', ni siquiera como término equivalente o sinónimo.

A partir de un sondeo hecho con hablantes en sus comunidades y en Tegucigalpa ${ }^{14}$, conducente a aclarar aspectos relacionados con la función y uso de estos dos términos, así como con sus respectivas asociaciones semánticas (connotaciones) y registros especializados si los hubiere - se pudieron obtener algunas referencias importantes:

- Carolina David reporta que en Trujillo y comunidades aledañas, el término canónico para VIH-SIDA es 'luriba sandi'; mientras 'gáduru-leta' prácticamente no se utiliza para designar esta enfermedad, aunque las personas entienden en general que se refiere a VIH-SIDA.

- Por otra parte, de diez jóvenes garínagu entrevistados en

${ }^{14}$ Este sondeo fue realizado durante el mes de agosto de 2009, por Carolina David en Trujillo, y por Salomé García y Johana Colindres con diez estudiantes garínagu de la UNAH, Tegucigalpa. 
Tegucigalpa (originarios de diferentes comunidades de los departamentos de Colón y Atlántida), todos reconocieron el término 'luriba sandi' como VIH-SIDA, mientras que solamente tres de ellos dijeron que 'gáduru-leta' se refiere a dicha enfermedad.

Una investigación exhaustiva de corte etno y sociolingüístico, podría identificar las diferencias entre los posibles usos metafóricos de estos dos términos, así como las razones detrás de la tendencia a la «oficialización» del término 'gáduru-leta', quizá por tratarse de uno más neutro.

\section{Conclusiones}

La revisión del vocabulario EGS, aunque se encuentra en cierne, permite plantear algunas observaciones preliminares que nuevas investigaciones podrán corroborar:

El pueblo garífuna, tanto sus organizaciones como las personas en su vida cotidiana, muestra una actitud favorable a la renovación de su patrimonio lingüístico aprovechando la potencialidad de su propia lengua. La investigación terminológica muestra que la lengua garífuna posee en efecto un gran potencial para hablar sobre la salud y la enfermedad, lo que se manifiesta en el hecho de que de 407 términos considerados de importancia por el proyecto de Unión Latina, sólo el 13\% debió ser aportado por transferencias directas (íntegras) provenientes del español mientras que el restante $87 \%$ pudo ser aportado mediante diversas estrategias desde los recursos de la propia lengua.

La consideración de los distintos fenómenos de transferencia léxica demuestra el dinamismo inherente al habla garífuna y su capacidad para responder a las situaciones del presente con creatividad y originalidad. No obstante, amerita una consideración más detenida la situación diglósica de los hablantes garínagu en la medida en que la alternancia con el español activa estrategias igualmente creativas (si bien enculturizadoras) frente a las situaciones prácticas. 
Esto supone que la política lingüística y la aspiración de los hablantes de renovar y mantener vigente su lengua, se encuentra en competencia con estas estrategias que llevan de su parte el ser realistas y efectivas para los objetivos prácticos inmediatos. La observación de algunos de los casos de transferencia léxica, como el referido a la designación del VIH/SIDA, apunta a la necesidad de reconsiderar las implicaciones socioculturales, por cuanto comprometen construcciones del universo cultural garífuna (enfermedad/mal) con las de la ciencia (virus/epidemia). 